\title{
Congenital heart disease and aortic arch variants associated with mutation in PHOX2B
}

\author{
Rachel C. Lombardo, MD ${ }^{1,2}$, Aleksey Porollo, $\mathrm{PhD}^{2,3}$, James F. Cnota, MD ${ }^{2,4}$ and \\ Robert J. Hopkin, $\mathrm{MD}^{1,2}$
}

Purpose: Congenital central hypoventilation syndrome (CCHS, OMIM 209880) is a rare autosomal dominant disorder caused by mutation in $P H O X 2 B$ that manifests as a consequence of abnormal neural crest cell migration during embryogenesis. Unlike other neurocristopathies, however, its impact on the cardiovascular system has not been previously assessed. This study was an effort to characterize the association between congenital heart disease (CHD) and mutations in PHOX2B in patients with CCHS.

Methods: A retrospective review of patients with CCHS in conjunction with functional analysis of $P H O X 2 B$ mutations associated with $\mathrm{CHD}$ was performed. To substantiate functional implications of identified variants, we conducted protein structure analyses and in silico mutagenesis were conducted.

Results: The prevalence of CHD among patients with CCHS was significantly greater $(30 \% ; p<0.001)$ than that of the current

\section{INTRODUCTION}

Congenital central hypoventilation syndrome (CCHS, OMIM 209880) is a rare autosomal dominant disorder characterized by central alveolar hypoventilation, Hirschsprung disease (HSCR), risk for tumors of neural crest cell origin, and risk for cardiac arrhythmias. It results from mutation in the gene PHOX2B, which encodes a homeodomain transcription factor critical for normal neural crest cell migration during embryogenesis. Mutant PHOX2B has been clearly documented to result in aberrant neural crest cell migration in both the brain and enteric nervous system. Failure of normal enteric neural crest cell migration ${ }^{1}$ and neuronal specification to the locus ceruleus ${ }^{2}$ results in two of the hallmarks of this disorder, Hirschsprung disease and decreased sensitivity to hypercapnia, respectively. Predisposition to development of tumors of the sympathetic nervous system, most commonly neuroblastoma, is also attributed to this phenomenon. ${ }^{3}$ The role PHOX2B may play with respect to cardiac neural crest cell migration and differentiation, however, has not been defined.

It is widely accepted that cardiac neural crest cell (cNCC) migration and differentiation is a critical component of normal development of the pharyngeal arch arteries, aortic arch, and cardiac outflow tract. ${ }^{4}$ Absent or reduced numbers of cNCCs migrating into the developing heart result in a wide estimated prevalence of CHD. The majority of patients had anomalies involving the proximal aortic arch and/or proximal coronary arteries. Variants associated with CHD in this cohort appear to disrupt DNA binding of PHOX2B via alteration of its homeobox domain.

Conclusion: This is the first report of an association between CHD and mutation in $P H O X 2 B$. Results are highly suggestive that alteration or elimination of the homeobox domain conveys significant risk for associated $\mathrm{CHD}$ or aortic arch variation.

Genet Med advance online publication 15 March 2018

Key Words: aortic arch; cardiac neural crest cell; congenital central hypoventilation syndrome; congenital heart disease; PHOX2B

range of cardiovascular defects including cotruncal anomalies and abnormalities as a result of erroneous remodeling of the pharyngeal arches. ${ }^{5-8}$ Previous studies examining cNCCderived mesenchymal cells in mammalian models have confirmed substantial contribution to the development of the pharyngeal arches, proximal aortic arch, ${ }^{9}$ proximal origins of the coronary arteries, the semilunar valves, and the atrioventricular valves, ${ }^{9-11}$ but the mechanisms regulating their migration are largely unknown.

We recently reported two patients with mutation in PHOX $2 B$ and congenital heart disease (CHD). ${ }^{12}$ There have been two additional reports of $\mathrm{CHD}$ in association with PHOX2B mutation, one patient with tetralogy of Fallot ${ }^{13}$ and a patient identified as having a ventricular septal defect. ${ }^{14}$ Therefore, to determine whether an association between CHD and mutation in $P H O X 2 B$ existed, a single-institution retrospective review was performed. The potential mechanisms in which mutant $P H O X 2 B$ may result in aberrant cNCC migration and differentiation are also discussed.

\section{MATERIALS AND METHODS}

A single-institution retrospective review of patients with CCHS evaluated at Cincinnati Children's Hospital and Medical Center from January 2003 to June 2016 was

${ }^{1}$ Division of Human Genetics, Cincinnati Children's Hospital and Medical Center, Cincinnati, Ohio, USA; ${ }^{2}$ Department of Pediatrics, University of Cincinnati College of Medicine, Cincinnati, Ohio, USA; ${ }^{3}$ Center for Autoimmune Genomics and Etiology, Cincinnati Children's Hospital and Medical Center, Cincinnati, Ohio, USA; ${ }^{4}$ Division of Cardiology, Cincinnati Children's Hospital and Medical Center, Cincinnati, Ohio, USA. Correspondence: Rachel C. Lombardo (rachel.lombardo@cchmc.org) 


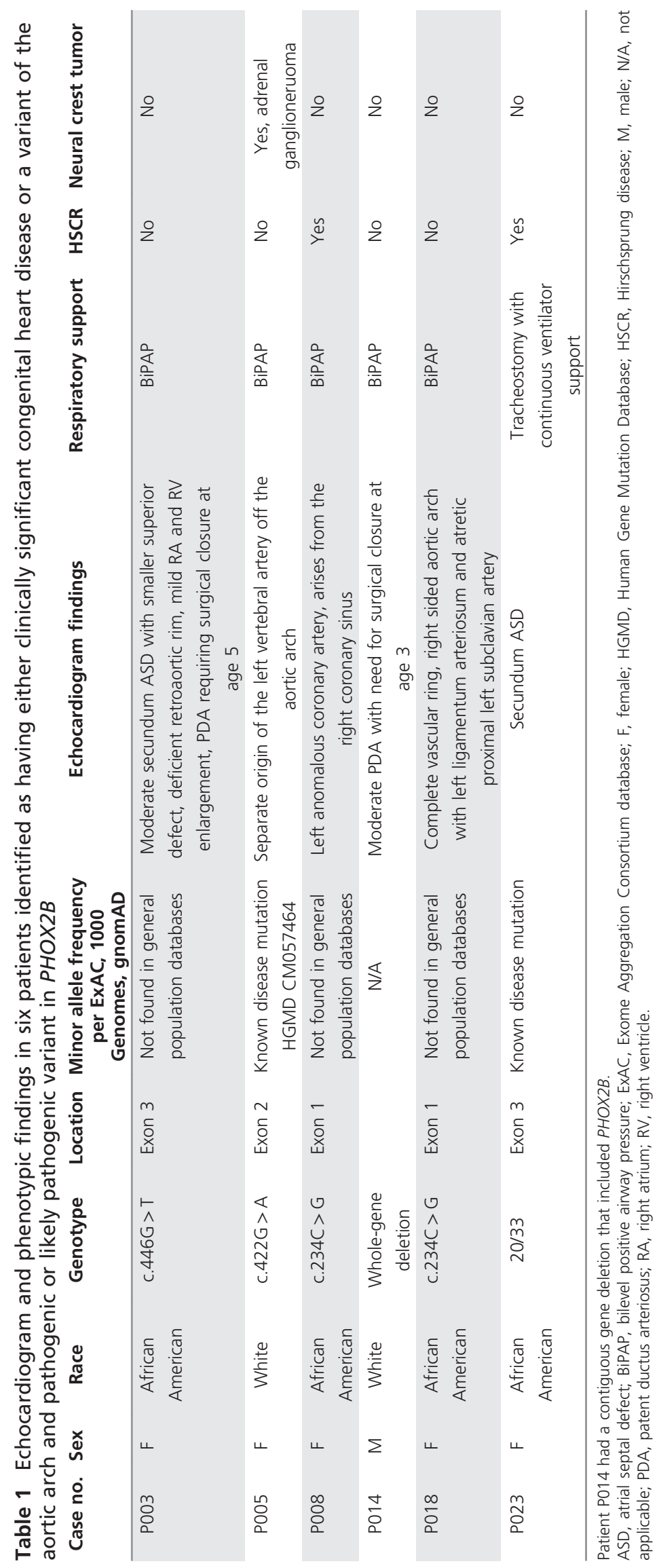


performed. The study was approved by the hospital's institutional review board prior to initiation. Electronic medical records were searched using the following search terms and accompanying International Classification of Diseases (9th revision) codes: central alveolar hypoventilation 348.8 , congenital central hypoventilation syndrome 327.25, CCHS 327.25, and central hypoventilation syndrome 327.25. All data was queried and extracted from the electronic health record system with manual extraction via chart review.

Inclusion criteria consisted of a diagnosis of CCHS based on the presence of central alveolar hypoventilation in the absence of primary pulmonary, cardiac, neuromuscular, or neurologic disease. Results of molecular analysis of the $P H O X 2 B$ gene, when available, were used for confirmation of clinical diagnosis. If molecular testing had not been performed, the presence of associated comorbidities such as autonomic dysfunction and Hirschsprung disease was considered supportive of diagnosis and indicative of inclusion in the study. Patients in whom a secondary cause of central alveolar hypoventilation was present or $P H O X 2 B$ sequencing was negative were excluded.

Data collected in individuals who met clinical criteria for diagnosis of CCHS included results of $P H O X 2 B$ sequencing, details of cardiac anatomy as reported by echocardiogram, electrocardiogram findings, and Holter monitor reports. All patients had received screening echocardiograms per the American Thoracic Society clinical policy statement due to risk for development of pulmonary hypertension. ${ }^{15}$ Clinical summary of echocardiogram findings was reviewed for all patients. Images were reviewed if study was reported as abnormal or if findings were ambiguous.

To determine significance of findings, a Fisher's exact twotailed test was performed comparing the prevalence of CHD in our cohort with the most recent estimates for the prevalence of CHD within the United States (6.9 per 1,000 live births) and worldwide (8 per 1,000 live births). ${ }^{16}$ Relative risk for patients with CCHS as compared with these two control populations was also calculated.

To substantiate functional implications of these mutations, a $3 \mathrm{D}$ model of the homeobox domain of human Phox $2 b$ protein was retrieved from the ModBase database. ${ }^{17}$ Protein structure analysis and in silico mutagenesis were conducted using PyMol.

\section{RESULTS}

Twenty-six patients who met the clinical criteria for diagnosis of CCHS were identified and had charts available for review (Supplementary Table S1 online); however, only 20 had undergone molecular analysis of $\mathrm{PHOX} 2 \mathrm{~B}$. Of those with molecular sequencing results, 11 had a polyalanine repeat mutation in exon 3 while 9 patients were identified as having a nonpolyalanine repeat mutation. One patient had a deletion on chromosome 4 that included the $P H O X 2 B$ gene. All $P H O X 2 B$ variants identified in patients with $\mathrm{CHD}$ were either known pathogenic mutations or did not have minor allele frequencies within the general population databases (Table 1).

Clinical findings are summarized in Table 1. Among patients with molecularly confirmed CCHS, 6 patients $(6 / 20$, $30 \%$ ) were identified as having clinically significant heart disease, and 14 patients had normal cardiac anatomy. One patient who met clinical criteria for CCHS based on polysomnography was identified as having diffuse chamber enlargement with aortic regurgitation at age 9. This was suggestive that an underlying defect may have been present leading to progressive ventricular and aortic valve enlargement. However, because molecular confirmation of $P H O X 2 B$ was not performed, this result was excluded from statistical analysis.

One patient was identified with a nonpolyalanine repeat mutation and an anomalous left vertebral artery arising directly from the aortic arch. Although this is considered a normal aortic arch variant, it is uncommon, with an estimated prevalence between 3 and $8 \%{ }^{18,19}$ in the general population. Five patients had anomalies involving the proximal aortic arch and/or proximal coronary arteries.

The calculated prevalence of CHD among patients with CCHS and molecular confirmation of mutation or deletion in PHOX $2 B$ at our institution was $30 \%(6 / 20, p<0.001)$. Relative risk for this population compared with the estimated prevalence of CHD in the United States was 42.9 (confidence interval 15.9-116.1, $p<0.001$ ); when compared with the worldwide estimate of 8 per 1,000 live births, relative risk was 37.5 (confidence interval 14.3-98.1, $p<0.001$ ).

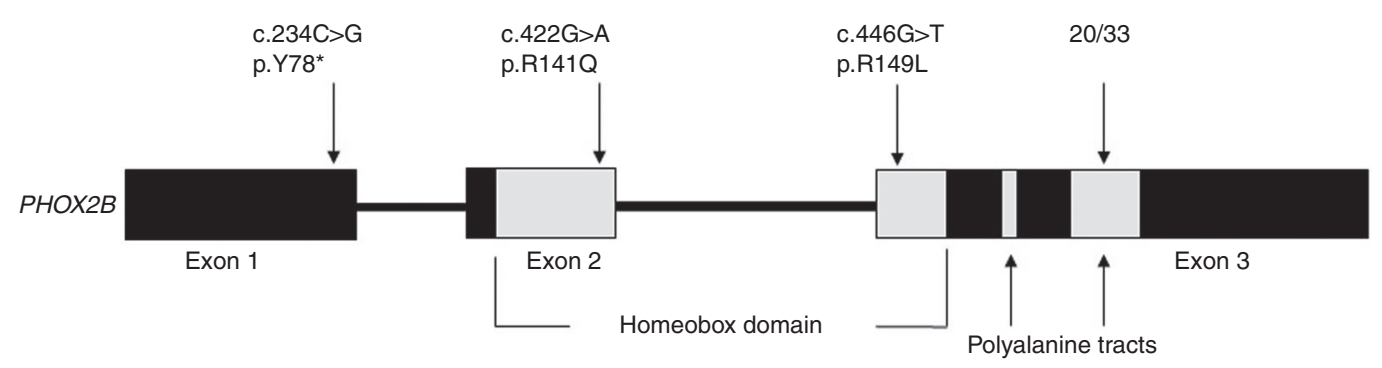

Figure 1 Approximate genomic location of $P H O X 2 B$ variants associated with congenital heart disease (CHD) and/or an aortic arch variant. Conserved arginine residues in the homeobox domain (positions 141 and 149) are also indicated. R141 is predicted to bind a phosphate group of nucleotides within target DNA motifs. R149 is predicted to be involved in 3D fold stabilization of the homeobox domain via electrostatic interaction with E114. 


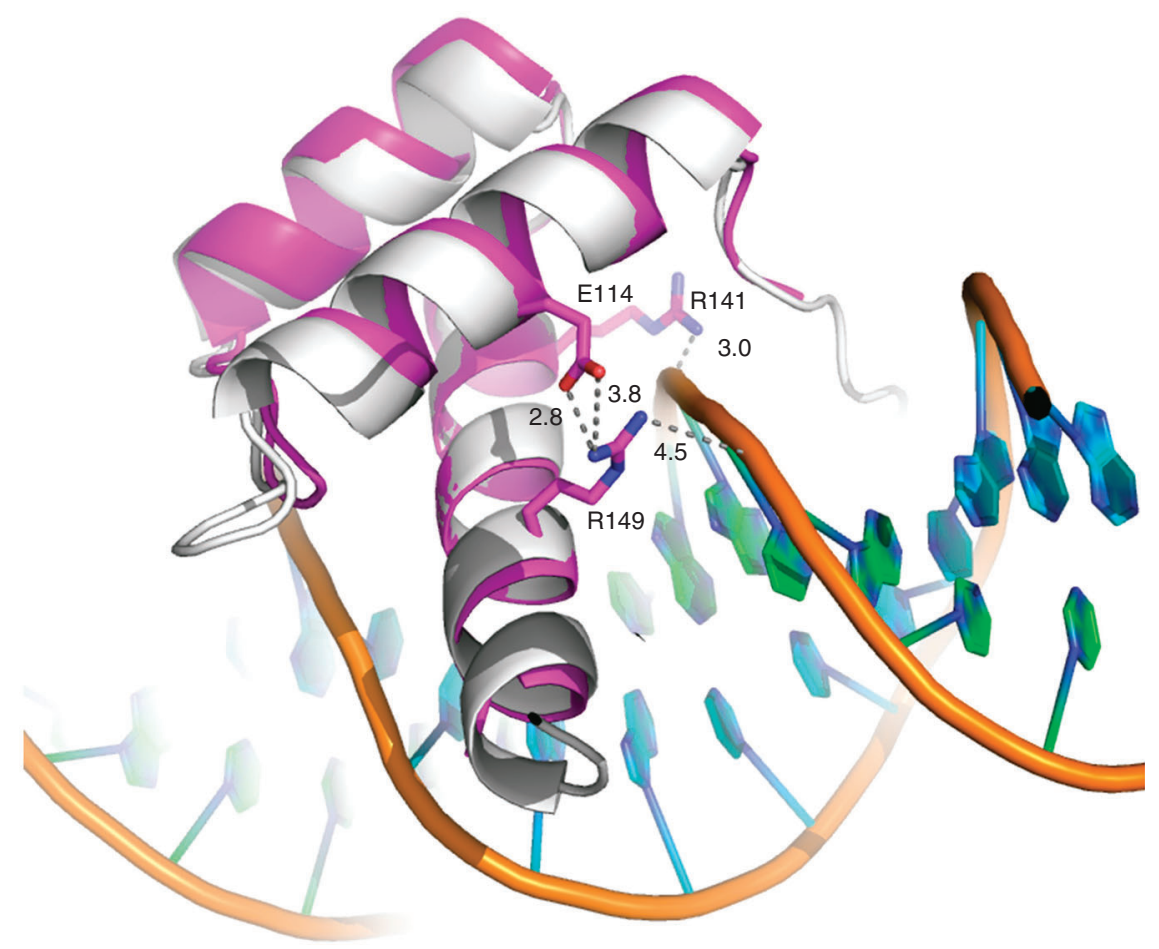

Figure 2 A 3D model of human PHOX2B homeobox domain (magenta) aligned to a resolved structure of the human PBX1 homeodomain (gray) cocrystallized with a DNA (PDB ID 1B72, chain B). Residues with identified point mutations (R141 and R149) are rendered as sticks with nitrogen (blue). Dashed lines and numbers indicate distances $(\AA)$ to the closest atoms: oxygen (red) of E114 and phosphorus (orange) in phosphate groups of the DNA backbone.

Table 2 Predicted functional consequence of mutation in $P H O X 2 B$ for those associated with congenital heart disease or aortic arch variation

\begin{tabular}{lll} 
DNA change & Amino acid change & Predicted functional consequence \\
\hline c.234C $>$ G & p.Y78* & Loss of homeobox domain due to premature stop codon \\
c. $422 \mathrm{G}>$ A & p.R141Q & Reduced DNA binding affinity to the target DNA motifs due to loss of R141-DNA backbone interaction \\
c. $446 G>T$ & p.R149L & Lost or reduced DNA binding affinity to the target DNA motifs due to homeodomain misfolding \\
$20 / 33$ & +13 Ala residues & Reduced ability to correctly activate target promoters \\
\hline
\end{tabular}

The 3D model of the PHOX2B homeobox domain, where both mutations p.R141Q and p.R149L are located (Figure 1), was aligned to a resolved structure of the human PBX1 homeodomain cocrystallized with a DNA (Protein Databank ID 1B72, chain B). The alignment resulted in root mean square deviation $1.844 \AA$ between 286 aligned nonhydrogen atoms, enabling the reliable localization of the mutated positions and their intra- and intermolecular contacts (Figure 2). Arg141 appears to be in contact with the phosphate group of the DNA backbone suggesting its role in the homeobox domain as a supporting residue that contributes to nonspecific binding affinity to DNA. Arg149 is in electrostatic interaction with Glu114 located at the opposite $\alpha$-helix of the same homeobox domain suggesting its primary role in the stabilization of $3 \mathrm{D}$ fold of the homeodomain. Possible functional consequences of identified mutations are summarized in Table 2.

\section{DISCUSSION}

Congenital heart disease, specifically anomalies involving the aortic arch and proximal coronary arteries, appears to be more common in patients with CCHS than the general population. The prevalence of CHD in our cohort was substantially higher than the estimated prevalence in both the United States and worldwide. All $P H O X 2 B$ variants associated with $\mathrm{CHD}$ in our cohort were either previously described pathogenic variants or absent from the general population databases (Table 1); therefore, it is unlikely that the observed association is secondary to rare ethnic variants. Our cohort exhibited a female predominance, but this is also unlikely to have contributed to our findings as several studies have demonstrated a male predominance in CHD. ${ }^{16,20-23}$ These findings are strongly suggestive that $P H O X 2 B$ plays a key role in the embryologic development of the heart and the identified variants are responsible for the observed anatomic changes. 
Aortic arch anomalies are believed to account for approximately one-fifth of all $\mathrm{CHD} ;{ }^{24}$ however, the proportion of involvement of the aortic arch and its derivatives in our cohort was much higher. The majority of patients with $\mathrm{CHD}$ and CCHS had anomalies involving the proximal aortic arch and/or proximal coronary arteries. An additional patient was identified as having an anomalous left vertebral artery arising directly from the aortic arch. Although this is considered a normal aortic arch variant, it is uncommon, with an estimated prevalence of $3-8 \%$ in the general population. ${ }^{18,19}$ The concentration of defects associated with the aortic arch and pharyngeal arch derivatives is likely directly related to their shared embryologic origin and dependence on migration of cNCCs. Prior studies have demonstrated mesenchymal cells within the ascending arch of the aorta, the ductus arteriosus and subsequent ligamentum arteriosum, ${ }^{9}$ the semilunar valves, and origins of the coronary arteries are cNCC derived. ${ }^{9}$ Jiang et al. ${ }^{9}$ also found that in aortic arch malformations, labeled neural crest cells could be identified in pharyngeal arch arteries that should have lost their investment of neural crest cells, indicating that the fate of the aortic arch arteries and cardiac neural crest cells are directly related.

Although there is no currently confirmed mechanism, CHD in CCHS may be the result of aberrant interaction between PHOX2B mutant protein, SOX10, and TWIST1. Twist1 has been found to play a critical role in cell fate in cardiac neural crest cells and is necessary for appropriate cNCC migration into the developing heart ${ }^{25}$ from the neural tube along proper pathways. ${ }^{26}$ During mesodermal cardiac neural crest cell differentiation, Twist1 and SOX10 have been demonstrated to bind to the $P H O X 2 B$ promoter to repress transcriptional activity. ${ }^{25}$ It is possible that mutation in the sequence of PHOX $2 B$ provides a mechanism for escape of downregulation of transcriptional activity. Further studies to assess the contribution of $P H O X 2 B$ to the migration of cardiac neural crest cells into the developing heart are needed.

Echocardiography is sufficient for diagnostic purposes in neonates and young children with CCHS; however, complete delineation of anomalies may require $3 \mathrm{D}$ imaging such as magnetic resonance imaging and computerized tomography. In patients in whom the aortic arch cannot be visualized, cross sectional imaging should be recommended. It is important to note, however, that ionizing radiation should be avoided in this population given predisposition to malignancy. ${ }^{27}$

Of the seven patients identified with CHD or an aortic arch variant, five had either a nonpolyalanine repeat mutation or whole-gene deletion (Table 1). It is still unclear how mutant PHOX2B exerts pathologic effect. Lascio et al. ${ }^{28}$ demonstrated decreased binding to target promoters in mutant PHOX2B with expanded alanine tracts. The variants associated with CHD in our cohort also appear to disrupt DNA binding via alteration of the homeobox domain (Table 2). Replacement of the arginine residue at position 141 to glutamine (p.R141Q) most likely reduces binding affinity of $P H O X 2 B$ to DNA. The p.R149L mutation disrupts electrostatic interaction between R149 and E114 thereby destabilizing a 3D fold of the homeobox domain and hence protein-DNA interaction whereas the p.Y78* variant lacks the entire homeodomain. These data are highly suggestive that nonpolyalanine repeat mutations that alter or eliminate the homeobox domain carry a high risk for associated CHD or aortic arch variation.

Potential target promoters of $P H O X 2 B$ in the developing heart are currently unknown. Interplay between PHOX2B, SOX10, and Twist1 in the mammalian heart has been demonstrated in prior experiments. ${ }^{25}$ Twistl has been found to play a critical role in cell fate in cardiac neural crest cells and is necessary for appropriate migration from the neural tube along proper cardiac neural crest cell pathways. ${ }^{26}$ During mesodermal cardiac neural crest cell differentiation, Twist1 and SOX10 are believed to bind to the PHOX2B promoter to repress transcriptional activity, ${ }^{25}$ but further studies are necessary to clarify this interaction.

There is a need for a large-scale, multicenter study to confirm these findings. In vitro studies to examine the PHOX $2 B$ expression pattern in the development of the mammalian heart, the associated cellular pathways, and mechanism leading to aberrant pharyngeal arch development are also necessary.

\section{SUPPLEMENTARY MATERIAL}

Supplementary material is linked to the online version of the paper at http://www.nature.com/gim

\section{DISCLOSURE}

The authors declare no conflict of interest.

\section{REFERENCES}

1. Carter TC, Kay DM, Browne ML, et al. Hirschsprung's disease and variants in genes that regulate enteric neural crest cell proliferation, migration and differentiation. J Hum Genet 2012;57:485-493.

2. Nobuta H, Cilio MR, Danhaive $O$, et al. Dysregulation of locus coeruleus development in congenital central hypoventilation syndrome. Acta Neuropathol 2015;130:171-183.

3. Trochet D, Bourdeaut F, Janoueix-Lerosey I, et al. Germline mutations of the paired-like homeobox 2B (PHOX2B) gene in neuroblastoma. Am J Hum Genet 2004;74:761-764.

4. Hutson MR \& Kirby ML. Neural crest and cardiovascular development: a 20-year perspective. Birth Defects Res C Embryo Today 2003;69:2-13.

5. Kirby $\mathrm{ML}$, Turnage $\mathrm{KL} \&$ Hays BM. Characterization of conotruncal malformations following ablation of "cardiac" neural crest. Anat Rec 1985;213:87-93.

6. Besson WT, Kirby ML, Van Mierop L \& Teabeaut JR. Effects of the size of lesions of the cardiac neural crest at various embryonic ages on incidence and type of cardiac defects. Circulation 1986;73:360-364.

7. Bockman DE, Redmond ME \& Kirby ML. Alteration of early vascular development after ablation of cranial neural crest. Anat Rec 1989;225: 209-217.

8. Conway SJ, Henderson DJ \& Copp AJ. Pax3 is required for cardiac neural crest migration in the mouse: evidence from the splotch $(\mathrm{Sp} 2 \mathrm{H})$ mutant. Development 1997;124:505-514

9. Jiang $X$, Rowitch $D H$, Soriano $P$, McMahon AP \& Sucov HM. Fate of the mammalian cardiac neural crest. Development 2000;127:1607-1616.

10. Poelmann R, Mikawa T \& Groot GD. Neural crest cells in outflow tract septation of the embryonic chicken heart: differentiation and apoptosis. Dev Dyn 1998;212:373-384.

11. Pietri $T$, Eder $O$, Blanche $M$, Thiery JP \& Dufour $S$. The human tissue plasminogen activator-Cre mouse: a new tool for targeting specifically neural crest cells and their derivatives in vivo. Dev Biol 2003;259:176-187.

12. Lombardo RC, Kramer E, Cnota JF, Sawnani H \& Hopkin RJ. Variable phenotype in a novel mutation in PHOX2B. Am J Med Genet A 2017;173: 1705-1709. 
13. Matera I, Bachetti T, Puppo F, et al. PHOX2B mutations and polyalanine expansions correlate with the severity of the respiratory phenotype and associated symptoms in both congenital and late onset central hypoventilation syndrome. J Med Genet 2004;41:373-380.

14. Shimokaze T, Sasaki A, Meguro T, et al. Genotype-phenotype relationship in Japanese patients with congenital central hypoventilation syndrome. J Hum Genet 2015;60:473-477.

15. Weese-Mayer DE, Berry-Kravis EM, Ceccherini I, et al. An official ATS clinical policy statement: congenital central hypoventilation syndrome: genetic basis, diagnosis, and management. Am J Respir Crit Care Med 2010;181:626-644.

16. van der Linde $D$, Konings $E E$, Slager $M A$, et al. Birth prevalence of congenital heart disease worldwide. J Am Coll Cardiol 2011;58: 2241-2247.

17. Pieper U, Webb BM, Dong GQ, et al. ModBase, a database of annotated comparative protein structure models and associated resources. Nucleic Acids Res 2014;42:D336-346.

18. Singla RK, Sharma $T \&$ Sachdeva K. Variant origin of left vertebral artery. Int J Anat Var 2010;3:97-99.

19. Jakanani $G \&$ Adair W. Frequency of variations in aortic arch anatomy depicted on multidetector CT. Clin Radiol 2010;65:481-487.

20. Nembhard WN, Wang T, Loscalzo ML \& Salemi JL. Variation in the prevalence of congenital heart defects by maternal race/ethnicity and infant sex. J Pediatr 2010:259-264.
21. Zhao QM, Ma XJ, Jia B \& Huang G. Prevalence of congenital heart disease at live birth: an accurate assessment by echocardiographic screening. Acta Paediatr 2013:397-402.

22. Reller MD, Strickland MJ, Riehle-Colaruso T, Mahle WT \& Correa A. Prevalence of congenital heart diefects in metropolitan Atlanta, 19982005. J Pediatr 2008:801-813.

23. Wu MH, Chen C, Lu CW, Wang JK, Huang SC \& Huang SK. Prevalence of congenital heart disease at live birth in Taiwan. J Pediatr 2010:782-785.

24. Goldmuntz E. The epidemiology and genetics of congenital heart disease. Clin Perinatol 2001;28:1-10.

25. Vincentz JW, Firulli BA, Lin A, Spicer DB, Howard MJ \& Firulli AB. Twist1 controls a cell-specification switch governing cell fate decisions within the cardiac neural crest. PLOS Genet 2013;9:e1003405.

26. Vincentz JW, Barnes RM, Rodgers R, Firulli BA, Conway SJ \& Firulli AB. An absence of Twist1 results in aberrant cardiac neural crest morphogenesis. Dev Biol 2008;320:131-139.

27. Berry-Kravis EM, Zhou L, Rand CM \& Weese-Mayer DE. Congenital central hypoventilation syndrome: $\mathrm{PHOX} 2 \mathrm{~B}$ mutations and phenotype. Am J Respir Crit Care Med 2006;174:1139-1144.

28. Di Lascio S, Bachetti T, Saba E, Ceccherini I, Benfante R \& Fornasari D. Transcriptional dysregulation and impairment of PHOX2B auto-regulatory mechanism induced by polyalanine expansion mutations associated with congenital central hypoventilation syndrome. Neurobiol Dis 2013;50: 187-200. 